Revista Brasileira de Cartografia

ISSN 1808-0936 | https://doi.org/10.14393/revbrascartogr

Sociedade Brasileira de Cartografia, Geodésia, Fotogrametria e Sensoriamento Remoto

\title{
Acurácia de Produtos Fotogramétricos Gerados com Aeronave Remotamente Pilotada em Relevo Acidentado
}

\section{Accuracy of Photogrammetric Products Generated by Remotely Piloted Aircrafts in Rough Reliefs}

Henrique Lopes Siqueira ${ }^{1}$, José Marcato Junior ${ }^{2}$, Edson Takashi Matsubara ${ }^{3}$, Reinaldo Almeida Colares ${ }^{4}$ e Fabio Martins Santos 5

1 Universidade Federal de Mato Grosso do Sul, FAENG, Campo Grande, Brasil. henrique.siqueira@ufms.br ORCID: https://orcid.org/0000-0001-8719-5901

2 Universidade Federal de Mato Grosso do Sul, FAENG, Campo Grande, Brasil.jrmarcato@ gmail.com

ORCID: https://orcid.org/0000-0002-9096-6866

3 Universidade Federal de Mato Grosso do Sul, FACOM, Campo Grande, Brasil. edsontm@facom.ufms.br

ORCID: https://orcid.org/0000-0002-4471-0886

4 HoursGeo, Belo Horizonte, Brasil. E-mail. reinaldo@ horusgeo.com.br

ORCID: https://orcid.org/0000-0002-6056-9931

5 HoursGeo, Belo Horizonte, Brasil. E-mail. fabio@ horusgeo.com.br

ORCID: https://orcid.org/0000-0002-2910-7077

Resumo: Apesar da popularização do uso de aeronaves remotamente pilotadas (RPA) como ferramentas para produção de produtos fotogramétricos e cartográficos digitais, pouco se fala a respeito da acurácia de tais produtos no contexto de terrenos acidentados, onde a diferença abrupta de altitudes gera uma maior complexidade na modelagem do relevo e consequentemente na geração das ortofotos. O objetivo desse trabalho é apresentar a avaliação e classificação baseado no PEC-PCD (Padrão de Exatidão Cartográfica para Produtos Cartográficos Digitais) de ortofotomosaicos e modelos digitais de superfície (DSM) gerados para uma mesma área de mineração. Para esse estudo utilizamos imagens RGB (não métrica) com GSD (Ground Sample Distance) estimado de 2,45 cm, e sobreposição de $80 \% / 80 \%$, captadas por RPA do tipo multirotor em dois voos idênticos realizados em datas distintas, para cada voo foram utilizados 15 alvos pré-sinalizados dos quais foram coletadas as coordenadas $\mathrm{X}$, Y e Z com auxilio de equipamento GNSS RTK. Cinco experimentos foram realizados, variando o número de GCP (Ground Control Points) e mantendo o número de CP (Check Points). Os produtos (ortofotomosaicos e DSM) gerados com as diferentes configurações de GCP, foram avaliados com base no PEC-PCD e, analisando os resultados obtidos foi possível constatar a variação de escala na qual os produtos se enquadram, esse fato foi atribuído à quantidade e disposição (geometria) dos GCP. De forma geral, os produtos gerados com 6 e 8 GCP apresentaram níveis de acurácia semelhantes entre si e foram classificados como Classe A para a escala 1:1000.

Palavras-chave: Fotogrametria. Ortofotomosaico Digital. RPA. Acurácia.

Abstract: Despite the popularization of the use of remotely piloted aircraft (RPA) as tools for the production of photogrammetric and digital cartographic products, little is said about the accuracy of such products in the context of rugged terrain, where the abrupt difference in altitudes leads to greater complexity. relief modeling and consequently in the generation of orthophotos. The aim of this paper is to present the evaluation and classification based on the PEC-PCD (Cartographic Accuracy Standard for Digital Cartographic Products) of orthophotomosaics and digital surface models (DSM) generated for the same mining area. For this study we used RGB (non-metric) images with GSD (Ground Sample Distance) estimated at $2.45 \mathrm{~cm}$, and $80 \%$ / 80\% overlap, captured by multirotor RPA on two identical flights performed on different dates, for each 15 pre-signaled targets were used from which the X, Y and Z coordinates were collected with the aid of GNSS RTK equipment. Five experiments were performed, varying the number of GCP (Ground Control Points) and maintaining the number of CP (Check Points). The products (orthophotomosaic and DSM) generated with the different GCP configurations were evaluated based on the PECPCD and, after the analysis of the results obtained, it was possible to verify the scale variation in which the products fit, this fact was attributed to the quantity and GCP arrangement (geometry). In general, products generated with 6 and 8 GCP presented similar accuracy levels and were classified as Class A for the 1: 1000 scale.

Keywords: Photogrammetry. Digital Orthophotomosaic. RPA. Accuracy. 


\section{INTRODUÇÃO}

O mercado da mineração, dentre outros muitos mercados que atuam na extração, processamento e/ou venda de matéria prima natural, possui uma constante demanda por mensurações de volumes e áreas em grande escala, sendo assim, a segurança, a praticidade e o tempo necessário para os levantamentos; os custos envolvidos com equipamentos; a precisão/acurácia e a velocidade na geração dos produtos e resultados, dentre possíveis outros fatores, são de extrema importância para a operação destes mercados.

As mensurações de grandes volumes com superfícies irregulares através de equipamentos de levantamento terrestre como receptores GNSS (Global Navigation Satellite System) e Estações Totais demandam grande quantidade de tempo e também custos. Para estes casos, uma alternativa a se considerar e o uso de Aeronaves Remotamente Pilotadas, ou Remotely Piloted Aicrafts (RPA).

\subsection{Justificativa do Estudo}

Devido a grande quantidade de pontos homólogos (nuvem de pontos) gerados após o processamento utilizando imagens de RPA, é possível extrair dos aerolevantamentos uma grande quantidade de informação e, além disso, esperam-se resultados de mensurações fidedignos.

A realização de medições indiretas também traz benefícios relacionados a segurança dos profissionais, evitando movimentações dos mesmos sobre pilhas de material e próximo a equipamentos e veículos de grande porte.

Porém, apesar de conhecidas as vantagens apresentadas pelo uso de RPA, muito ainda se discute a respeito do impacto de alguns parâmetros na acurácia dos produtos gerados, por exemplo a quantidade de GCP (Ground Control Poins), quantidade de CP (Check Points), o grau de sobreposição das imagens (Overlap), a altitude de voo (Flight Altitude), e consequentemente o GSD (Ground Sample Distance) da imagem, entre outros.

\subsection{Estado da Arte e Objetivo}

De acordo com Westoby et al. (2012) deve-se tomar um cuidado especial com relação ao estabelecimento das redes de GCP, visando facilitar a transformação dos pontos para um sistema de coordenadas absolutas e a extração de dados métricos, a fim de obter resultados acurados. Apesar disso, os autores enfatizam a viabilidade do uso dessa metodologia como alternativa as técnicas de levantamento topográfico tradicionais.

Quanto maior o número de GCP utilizado, maior o trabalho de campo relativo a aquisição de dados com alvos em solo. Sendo assim é desejável encontrar um valor mínimo para o número de GCP, que atenda as exigências relacionadas a acurácia e garantindo o mínimo possível de trabalho de campo.

James et al. (2017) apontam que, em alguns casos, é possível reduzir o número de GCP utilizados em levantamentos com RPA pela metade, com o mínimo de impacto na qualidade do produto cartográfico final.

Até o momento não existe um método universal para estabelecer a quantidade de GCP necessária para garantir um determinado grau de acurácia. Sendo assim, é essencial o desenvolvimento de pesquisas nessa área, visando aprofundar o nível de conhecimento técnico e científico.

Também é necessária atenção especial as normativas relativas aos produtos cartográficos digitais. No Brasil adota-se o PEC-PCD (Padrão de Exatidão Cartográfica para Produtos Cartográficos Digitais), o qual é definido na Especificação Técnica para Aquisição de Dados Geoespaciais Vetoriais (ET-ADGV) EB10-N72.001 (BRASIL, 2012).

Zanetti et al. (2017) investigaram a influência de GCP em ortofotos geradas por imagens obtidas com RPA do tipo multirotor. Os autores realizaram testes com 5, 10 e 15 GCP bem distribuídos, além de um outro teste com 15 GCP concentrados, em uma área de 16,30 ha. Obtiveram valores de acurácia compatíveis com aplicação em escala 1:1000, porém variando sua classificação entre as classes A e D (de acordo com a PECPCD do ET-CQDG), dependendo diretamente do número de GCP utilizado no processamento. 
Alves Junior et al. (2018) realizaram um estudo semelhante, utilizando uma aeronave do tipo asa fixa. Os autores sobrevoaram uma área de 585 ha capturando imagens aéreas com GSD de $0,113 \mathrm{~m}$, utilizando 0,4 , 8 e 16 GCP. Os mapas produzidos foram classificados com erro planimétrico < 0,13m e erro Altimétrico < 0,30m. Apesar dos resultados apresentarem produtos com alta resolução, os autores apontaram que o uso de RPA modelo asa fixa torna o plano de voo suscetível a variações pelo deslocamento lateral da aeronave dependendo da atuação do vento, com isso o traçado de voo pode ser alterado causando distorções nas imagens. A altitude de voo da aeronave também apresentou variação neste estudo, o que ocasiona variação do GSD ao longo do voo. Os autores recomendaram a realização de estudos futuros para verificar a precisão do DSM, a distribuição e a quantidade mínima de GCP em função do GSD.

Além dos trabalhos mencionados anteriormente, outros trabalhos abordaram a avaliação de produtos cartográficos gerados com RPA como Krasak et al. (2016), Raczynski (2017), Molnar e Domozi. (2018), Oliveira et al. (2017), Costa e Silva. (2009), Gindraux et al (2018) e Zanetti et al. (2016). No entanto, não foram encontrados trabalhos onde se aborda a avaliação de produtos cartográficos gerados em relevos acidentados de acordo com as Normas Brasileiras.

Em relevos acidentados, ao realizar-se a calibração, tem-se como vantagem a redução de correlação entre POI (Parâmetros de Orientação Interior) e POE (Parâmetros de Orientação Exterior) (DEBIASI MITISHITA, 2013; CAMPOS et al., 2015). Como desvantagem, tem-se uma alta variabilidade do GSD em relevos acidentados (NADOLINETS et al., 2017).

Nesse contexto, o objetivo desse trabalho consiste em apresentar a avaliação e classificação de produtos cartográficos (Ortofotomosaico e modelos digitais de superfície) gerados para uma área de mineração baseado no PEC-PCD.

\section{METODOLOGIA}

Visando adequar o estudo ao modo de operação de profissionais da área, estabelecemos uma metodologia simples, baseada na captação de imagens aéreas com um RPA, seguido do processamento destas e por último uma classificação com relação a sua escala baseado nos critérios da ET-ADGV.

\subsection{Obtenção de Dados}

Para a realização deste trabalho foram realizados dois voos semelhantes, ambos sobre uma mesma região com área de 0,51 há. Esta área apresenta relevo acidentado, em decorrência de trabalhos de corte e movimentação de terras, com variação de aproximadamente 90 metros entre o ponto mais alto e o ponto mais baixo. O primeiro voo foi realizado em 2 de janeiro de 2018 e o segundo em 31 de janeiro de 2018.

A captura das imagens de ambos os voos foi realizada através de uma mesma RPA do tipo multirotor, modelo Phantom4 Pro, acoplado com uma câmera RGB de resolução 20MP (especificações na Tabela 1).

Tabela 1 - Especificações da câmera utilizada.

\begin{tabular}{l|l|l|l}
\hline Aeronave & Sensor & Lente & Resolução da imagem \\
\hline Phantom4 Pro & $1^{\prime \prime C M O S ~}$ & FOV $=84^{\circ} 8.8 \mathrm{~mm} / 24 \mathrm{~mm}$ & $5472 \times 3648(20 \mathrm{Mp})$ \\
\hline \multicolumn{2}{|c|}{ Fonte: DJI $(2017)}$.
\end{tabular}

O plano de voo utilizado foi elaborado em um aplicativo para dispositivo móvel, sendo ele dividido em 3 etapas devido a autonomia de voo do RPA. As características do plano de voo podem ser observadas nas Figuras (1 e 2). 
Figura 1 - Plano de Voo.

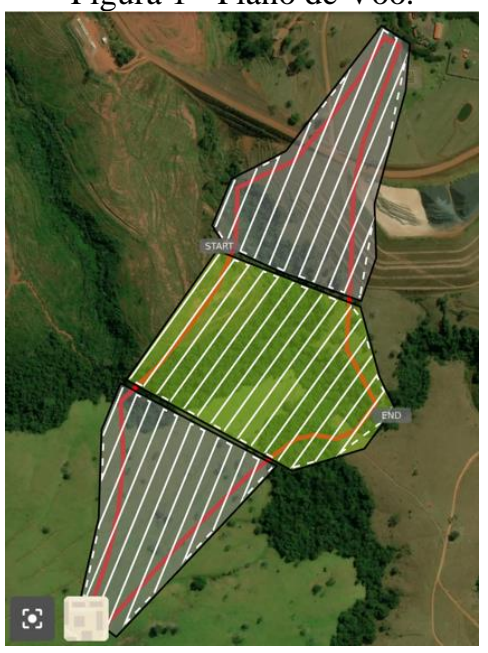

Figura 2 - Detalhes do Plano de Voo.

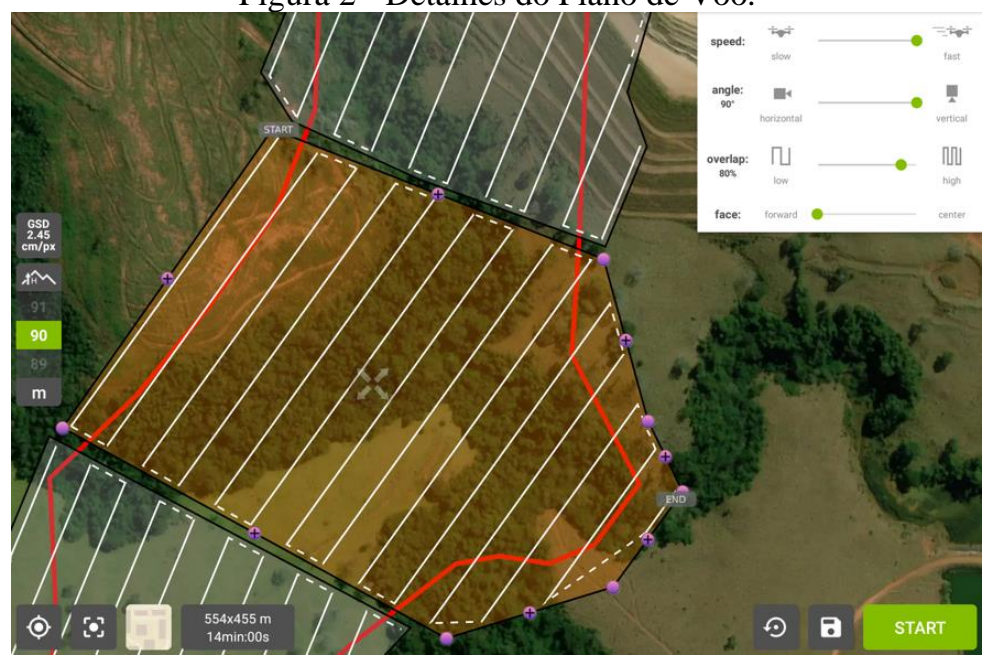

Fonte: Os autores (2020).

Para cada um dos voos foram capturadas imagens aéreas com sobreposições lateral e frontal de $80 \%$ e altura de voo de $90 \mathrm{~m}$ com relação ao ponto de decolagem, resultando em um GSD médio estimado de $2,45 \mathrm{~cm}$ (Tabela 2). Cabe ressaltar que para ambos os voos, a altitude do RPA com relação ao solo variou de acordo com o relevo do terreno, dessa forma alterando a real resolução dos pixels (GSD) das imagens.

Tabela 2 - Configurações dos Voos realizados. GSD estimado pelo aplicativo Pix4d Capture, de acordo com a altura de

\begin{tabular}{c|c|c|c}
\multicolumn{5}{c}{ voo inicial. } \\
\hline Área sobrevoada & Grau de sobreposição & Altura de voo & GSD * \\
\hline $0.51 \mathrm{~km}^{2}$ & $80 \% / 80 \%$ (lateral/frontal) & $90 \mathrm{~m}$ & $2,45 \mathrm{~cm}$ \\
\hline
\end{tabular}

Fonte: Os autores (2020).

Alvos pré-sinalizados foram posicionados estrategicamente na área de estudo, distribuídos de forma homogênea com parte deles nos bordos e parte no centro, para posterior utilização como GCP e CP (Check Points), as coordenadas de tais alvos foram levantadas in loco utilizando um receptor GNSS do tipo RTK, modelo Trimble R4 com precisão 3D de aproximadamente 5mm (levantamento GNSS estático horizontal na ordem de $3 \mathrm{~mm}+0,1 \mathrm{ppm}$ e vertical de $3,5 \mathrm{~mm}+0,4 \mathrm{ppm}-$ Trimble, 2013). O posicionamento dos alvos na área estudada, de acordo com sua identificação, pode ser visualizado nas das Figuras 3 e 4.

Como também pode ser observado pelas Figuras 3 e 4, a distribuição dos GCP para cada caso $(0,2,4$, 6 e 8 GCP) foi diferente do Voo A para o Voo B. Sendo assim possível analisar, para dois casos com mesmo número de GCP, se os produtos gerados para cada voo também terão níveis de acurácia semelhantes ou distintos.

Figura 3 - Identificação dos CP (esquerda) e GCP (direita) para o voo A.

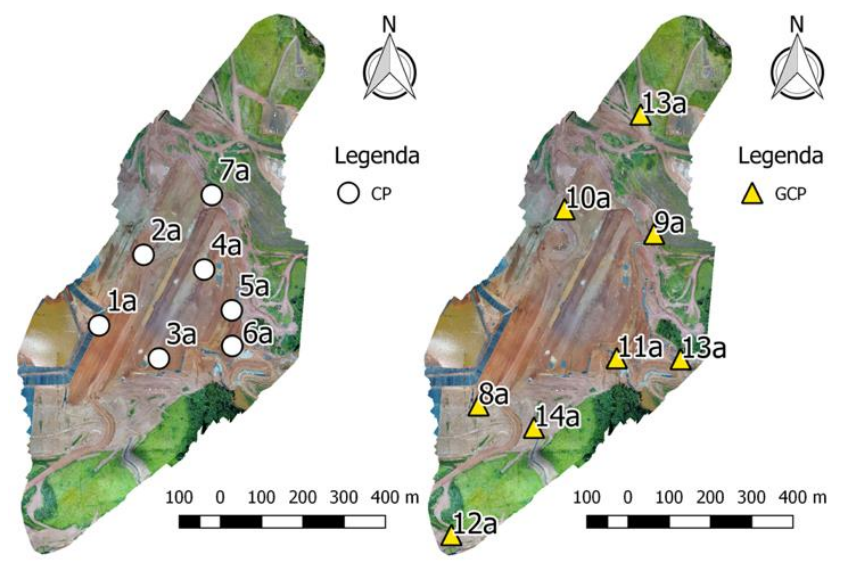

Fonte: Os autores (2020).
Figura 4 - Identificação dos CP (esquerda) e GCP (direita) para o voo B.

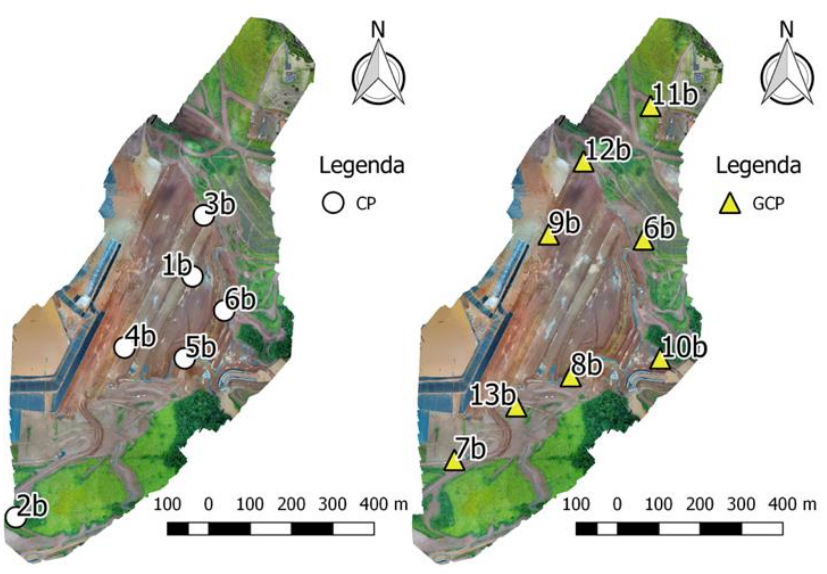

Fonte: Os autores (2020). 


\subsection{Processamento de Imagens}

As imagens foram processadas utilizando o software Pix4Dmapper (v.4.1.23) que combina fotogrametria e algoritmos de visão computacional, utilizando a metodologia SfM (Structure from Motion) para transformar imagens em ortomosaico e DSM.

Para cada um dos voos foram realizados 5 processamentos de imagem utilizando $0,2,4,6$ e 8 GCP (ver Figuras 5 e 6), gerando assim um total de 8 ortomosaicos, 2 mosaicos não controlados (0 GCP) (ver exemplo na Figura 7) e 10 DSM (ver exemplo na Figura 8). Após o processamento verificou-se uma densidade média de 16.5 pontos $/ \mathrm{m}^{3}$ para a nuvem de pontos e uma resolução de 1 xGSD $(2,91 \mathrm{~cm})$ para os ortomosaicos e DSM.

Figura 5 - Distribuição dos GCP e CP para cada processamento (Voo A).

Legenda: $\mathrm{GCP}=$ Triângulos Amarelos, $\mathrm{CP}=$ Círculos Brancos

(a) $0 \mathrm{GCP}+7 \mathrm{CP}$, (b) $2 \mathrm{GCP}+7 \mathrm{CP}$, (c) $4 \mathrm{GCP}+7 \mathrm{CP}$, (d) $6 \mathrm{GCP}+7 \mathrm{CP}$, (e) $8 \mathrm{GCP}+7 \mathrm{CP}$

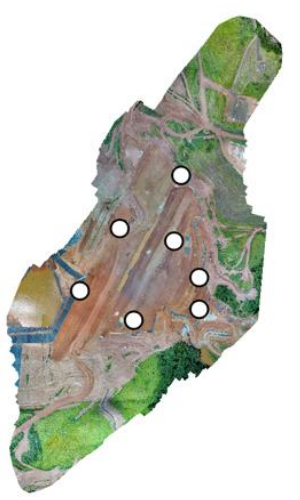

(a)

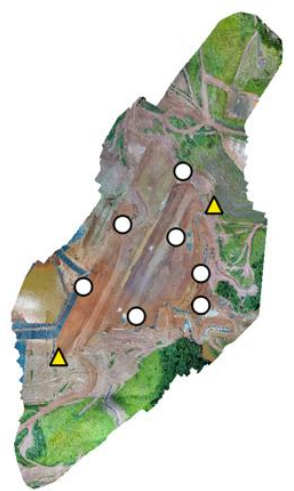

(b)



(c)

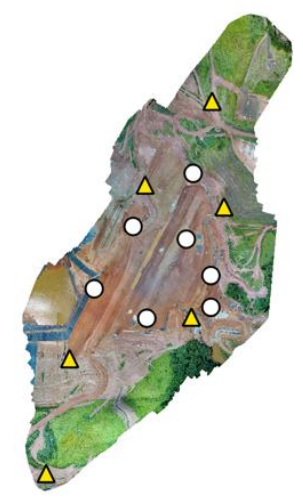

(d)

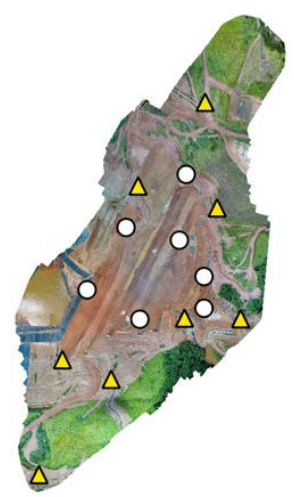

(e)

Fonte: Os autores (2020).

Figura 6 - Distribuição dos GCP e CP para cada processamento (Voo B).

Legenda: $\mathrm{GCP}=$ Triângulos Amarelos, $\mathrm{CP}=$ Círculos Brancos

(a) $0 \mathrm{GCP}+6 \mathrm{CP}$, (b) $2 \mathrm{GCP}+6 \mathrm{CP}$, (c) $4 \mathrm{GCP}+6 \mathrm{CP}$, (d) $6 \mathrm{GCP}+6 \mathrm{CP}$, (e) $8 \mathrm{GCP}+6 \mathrm{CP}$

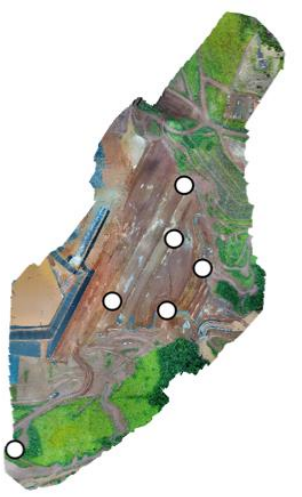

(a)

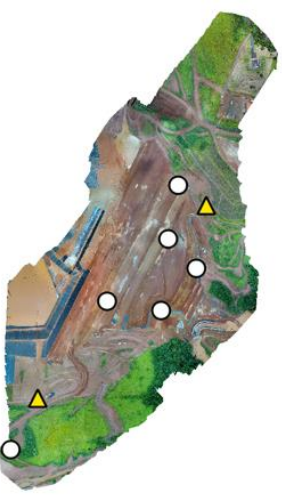

(b)

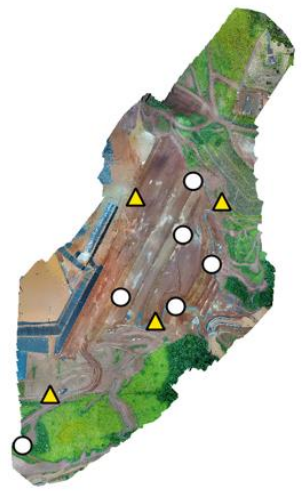

(c)

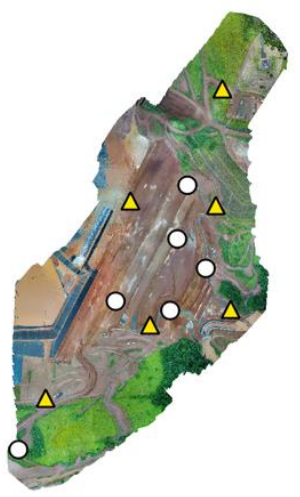

(d)

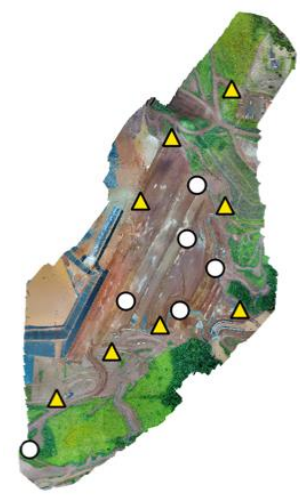

(e)

Fonte: Os autores (2020). 
Figura 7 - Exemplo de DSM gerado.

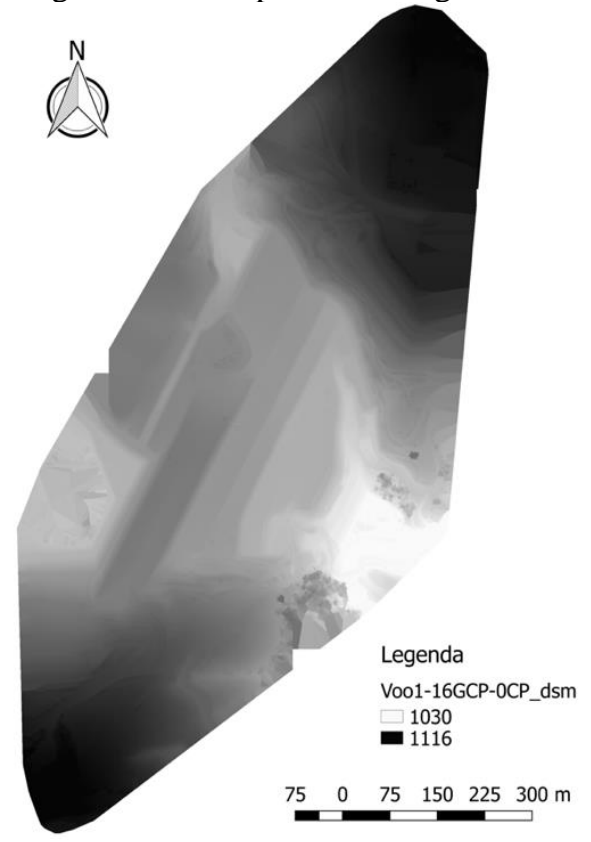

Fonte: Os autores (2020).
Figura 8 - Exemplo de Ortomosaicos gerado.

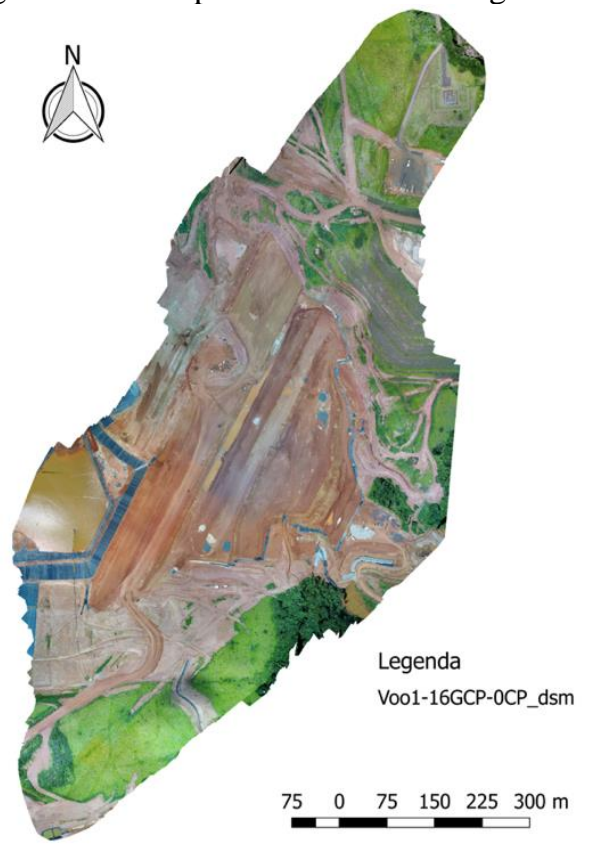

Fonte: Os autores (2020).

\subsection{Cálculo da Acurácia e Classificação do Produto Cartográfico Digital}

Para fins de avaliação do ortomosaico e DSM, as coordenadas dos CP estimadas no processamento das imagens (obtidas a partir do ortomosaico e do DSM) foram comparadas com as coordenadas dos alvos présinalizados levantados em campo com o RTK. As coordenadas geográficas (E, N e H) de cada CP foram obtidas através do software QGIS (QGIS DEVELPMENT TEAM, 2018), sendo referenciadas de acordo com o DATUM SIRGAS2000 / UTM 23S. As discrepâncias planimétricas e altimétricas (considerando altitude elipsoidal) foram então calculadas de acordo com a Eq. (1) e a Eq. (2), para posteriormente serem analisadas de acordo com os valores estabelecidos pelo PEC-PCD (Padrão de Exatidão Cartográfica para Produtos Cartográficos Digitais) e pelo EP (Erro Padrão), apresentados na Tabela 3.

$$
\begin{gathered}
\text { DiscrepânciaPlanimétrica }=\Delta S=\sqrt{\left(X_{i}-X_{r t k}\right)^{2}+\left(Y_{i}-Y_{r t k}\right)^{2}} \\
\text { DiscrepânciaAltimétrica }=\Delta H=Z_{i}-Z_{r t k}
\end{gathered}
$$

onde: $X_{i}=$ coordenada Leste do ponto obtida pelo ortomosaico; $X_{r t k}=$ coordenada Leste do ponto obtida pelo RTK; $Y_{i}=$ coordenada Norte do ponto obtida pelo ortomosaico; $Y_{r t k}=$ coordenada Norte do ponto obtida pelo RTK; $Z_{i}=$ altitude do ponto obtida pelo ortomosaico; e $Z_{r t k}=$ altitude Norte do ponto obtida pelo RTK.

Tabela 3 - Valores estabelecidos para a acurácia mínima de produtos cartográficos digitais, de acordo com o critério analisado (PEC-PCD / EP) e a escala a ser utilizada. *90\% dos pontos de verificação devem possuir discrepância posicional menor ou igual ao valor determinado pelo PEC. **o valor do RMSE (Root Mean Square Error) deve ser

\begin{tabular}{|c|c|c|c|c|}
\hline \multirow{3}{*}{$\begin{array}{c}\text { Acurácia } \\
\text { Mínima } \\
\text { Método } \\
\text { Escala (classe } \\
\text { A) }\end{array}$} & \multicolumn{2}{|c|}{ PEC-PCD } & \multicolumn{2}{|c|}{$\mathbf{E P}$} \\
\hline & \multicolumn{2}{|c|}{ PEC $\geq 90 \%$ das discrepâncias* } & \multicolumn{2}{|c|}{$\mathbf{E P} \geq \mathbf{R M S E} * *$} \\
\hline & Planimétrico & Altimétrico & Planimétrico & Altimétrico \\
\hline $1 / 10.000$ & $2,80 \mathrm{~m}$ & $1,35 \mathrm{~m}$ & $1,70 \mathrm{~m}$ & $0,84 \mathrm{~m}$ \\
\hline $1 / 5.000$ & $1,40 \mathrm{~m}$ & $0,54 \mathrm{~m}$ & $0,85 \mathrm{~m}$ & $0,34 \mathrm{~m}$ \\
\hline $1 / 2.000$ & $0,56 \mathrm{~m}$ & $0,27 \mathrm{~m}$ & $0,34 \mathrm{~m}$ & $0,17 \mathrm{~m}$ \\
\hline $1 / 1.000$ & $0,28 \mathrm{~m}$ & $0,27 \mathrm{~m}$ & $0,17 \mathrm{~m}$ & $0,17 \mathrm{~m}$ \\
\hline
\end{tabular}
menor ou igual ao valor determinado pelo EP.

Fonte: Adaptada de Brasil (2012). 
Por fim, foi realizada a classificação da escala dos produtos gerados para cada um dos voos, de acordo com o critério menos rigoroso (menor escala) para que fossem respeitadas tanto o critério do PEC-PCD quanto do EP.

Como foram utilizados 7 CP para o Voo A e 6 CP para o Voo B (ambos valores inferiores a 10), a verificação do critério de $90 \%$ dos valores passou a consistir em analisar apenas o valor das maiores discrepâncias planimétricas e altimétricas, comparando-as com o valor limite estabelecido pela PEC.

\section{RESULTADOS}

Os resultados obtidos para as divergências de posição (planimétrica e altimétrica) entre os pontos medidos com RTK e os pontos medidos através dos produtos gerados (ortomosaico e DSM), juntamente com os valores do RMSE, para os voos A e B são expostos pelas Tabelas 4 e 5.

Tabela 4 - Coordenadas E, N e H (X, Y e Z) obtidas pelos produtos gerados (ortomosaico e DSM) para o Voo A.

\begin{tabular}{|c|c|c|c|c|c|c|}
\hline $\mathbf{C P}$ & $\Delta \mathbf{S}(\mathbf{m})$ & $\Delta \mathbf{H}(\mathbf{m})$ & \multirow{9}{*}{ 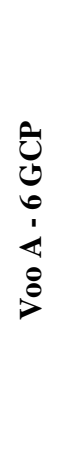 } & $\mathbf{C P}$ & $\Delta \mathbf{S}(\mathbf{m})$ & $\Delta \mathbf{H}(\mathbf{m})$ \\
\hline $1 \mathrm{a}$ & 0,625 & 32,641 & & $1 \mathrm{a}$ & 0,122 & $-0,103$ \\
\hline $2 \mathrm{a}$ & 0,449 & 30,368 & & $2 a$ & 0,106 & $-0,052$ \\
\hline $3 a$ & 0,941 & 34,213 & & $3 a$ & 0,063 & $-0,059$ \\
\hline $4 a$ & 1,086 & 31,396 & & $4 a$ & 0,068 & $-0,093$ \\
\hline $5 a$ & 1,377 & 33,182 & & $5 a$ & 0,052 & $-0,020$ \\
\hline $6 a$ & 1,533 & 34,519 & & $6 a$ & 0,059 & 0,062 \\
\hline $7 \mathrm{a}$ & 1,468 & 29,051 & & $7 \mathrm{a}$ & 0,106 & 0,036 \\
\hline RMSE & 1,138 & 32,250 & & RMSE & 0,086 & 0,067 \\
\hline $\mathbf{C P}$ & $\Delta \mathbf{S}(\mathbf{m})$ & $\Delta \mathbf{H}(\mathrm{m})$ & \multirow{18}{*}{$\begin{array}{l}0 \\
0 \\
\infty \\
1 \\
1 \\
8 \\
8\end{array}$} & $\mathbf{C P}$ & $\Delta \mathbf{S}(\mathbf{m})$ & $\Delta \mathbf{H}(\mathrm{m})$ \\
\hline $1 \mathrm{a}$ & 0,046 & $-2,314$ & & $1 \mathrm{a}$ & 0,135 & $-0,064$ \\
\hline $2 \mathrm{a}$ & 0,116 & $-2,145$ & & $2 a$ & 0,118 & $-0,037$ \\
\hline $3 a$ & 0,137 & $-0,696$ & & $3 a$ & 0,025 & $-0,064$ \\
\hline $4 a$ & 0,316 & $-2,506$ & & $4 a$ & 0,057 & $-0,023$ \\
\hline $5 a$ & 0,454 & $-2,835$ & & $5 a$ & 0,063 & 0,055 \\
\hline $6 a$ & 0,591 & $-2,943$ & & $6 a$ & 0,031 & 0,044 \\
\hline $7 \mathrm{a}$ & 0,115 & $-1,259$ & & $7 a$ & 0,119 & 0,021 \\
\hline RMSE & 0,317 & 2,236 & & RMSE & 0,089 & 0,047 \\
\hline $\mathbf{C P}$ & $\Delta \mathbf{S}(\mathbf{m})$ & $\Delta \mathbf{H}(\mathbf{m})$ & & & & \\
\hline $1 \mathrm{a}$ & 0,145 & $-0,268$ & & & & \\
\hline $2 \mathrm{a}$ & 0,107 & $-0,186$ & & & & \\
\hline $3 a$ & 0,082 & $-0,221$ & & & & \\
\hline $4 a$ & 0,060 & $-0,178$ & & & & \\
\hline $5 a$ & 0,056 & 0,005 & & & & \\
\hline $6 a$ & 0,063 & 0,173 & & & & \\
\hline $7 \mathrm{a}$ & 0,142 & 0,093 & & & & \\
\hline RMSE & 0,100 & 0,180 & & & & \\
\hline
\end{tabular}

Fonte: Os autores (2020). 
Tabela 5- Coordenadas E, N e H (X, Y e Z) obtidas pelos produtos gerados (ortomosaico e DSM) para o Voo B.

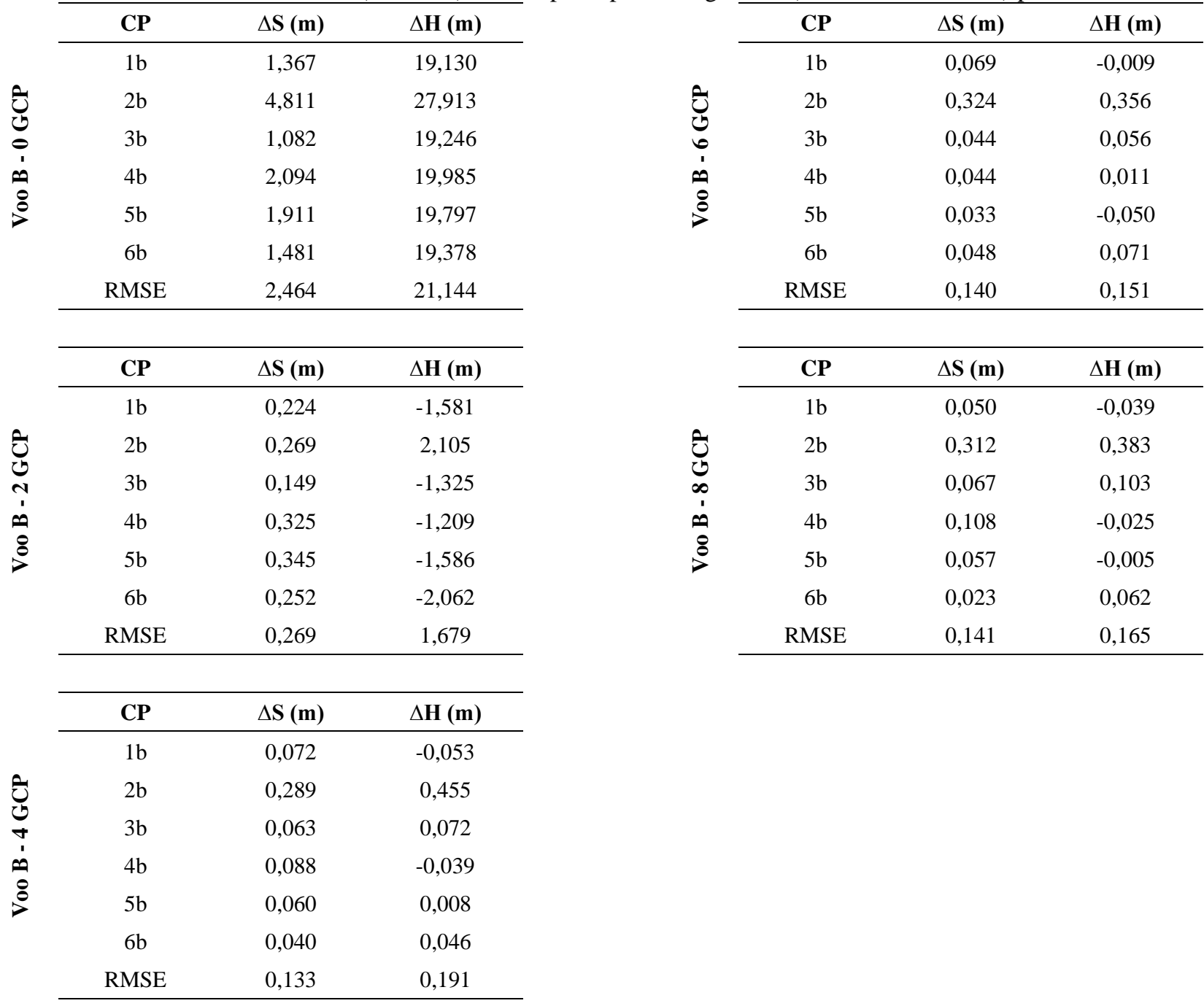

Fonte: Os autores (2020).

Os valores das discrepâncias máximas (planimétrica $=\Delta \mathrm{S}_{\max }$, altimétrica $=\Delta \mathrm{H}_{\max }$ ) e do RMSE de cada caso $(0,2,4,6$ e 8 GCP), foram então enumerados (Tabela 6), em seguida foram classificados de acordo com a ET-ADGV (Tabela 7).

Tabela 6 - Valores encontrados para as maiores discrepâncias (planimétrica e altimétrica) e RMSE de acordo com o número de GCP utilizados no processamento de cada caso.

\begin{tabular}{ccccc|cccc} 
& \multicolumn{4}{c}{ Voo A } & \multicolumn{4}{c}{ Voo B } \\
\hline Erro & \multicolumn{2}{c}{ Planimétrico $(\mathbf{m})$} & \multicolumn{2}{c|}{ Altimétrico $(\mathbf{m})$} & \multicolumn{2}{c}{ Planimétrico $(\mathbf{m})$} & \multicolumn{2}{c}{ Altimétrico $(\mathbf{m})$} \\
\hline Num. GCP & $\Delta \mathbf{S}_{\mathbf{m a x}}$ & $\mathbf{R M S E}$ & $\Delta \mathbf{H}_{\max }$ & $\mathbf{R M S E}$ & $\Delta \mathbf{S}_{\mathbf{m a x}}$ & $\mathbf{R M S E}$ & $\Delta \mathbf{H}_{\max }$ & $\mathbf{R M S E}$ \\
\hline 0 & 1,533 & 1,138 & 34,519 & 32,250 & 4,811 & 2,464 & 27,913 & 21,144 \\
2 & 0,591 & 0,317 & $-2,943$ & 2,236 & 0,345 & 0,269 & 2,105 & 1,679 \\
4 & 0,145 & 0,100 & $-0,268$ & 0,180 & 0,289 & 0,133 & 0,455 & 0,191 \\
6 & 0,122 & 0,086 & $-0,103$ & 0,067 & 0,324 & 0,140 & 0,356 & 0,151 \\
8 & 0,135 & 0,089 & $-0,064$ & 0,047 & 0,312 & 0,141 & 0,383 & 0,165 \\
\hline
\end{tabular}

Fonte: Os autores (2020). 
Tabela 7 - Classificação com base nos parâmetros PEC-EP para Escalas "Classe A”, de acordo com o número de GCP utilizados no processamento de cada caso.

\begin{tabular}{|c|c|c|c|c|c|c|c|c|c|}
\hline \multirow{3}{*}{\multicolumn{2}{|c|}{ Escala }} & \multicolumn{4}{|c|}{ Voo A } & \multicolumn{4}{|c|}{ Voo B } \\
\hline & & \multicolumn{2}{|c|}{ Planimétrica } & \multicolumn{2}{|c|}{ Altimétrica } & \multicolumn{2}{|c|}{ Planimétrica } & \multicolumn{2}{|c|}{ Altimétrica } \\
\hline & & PEC & EP & PEC & EP & PEC & EP & PEC & EP \\
\hline \multirow{5}{*}{ 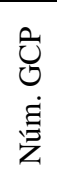 } & 0 & $1 / 10.000$ & $1 / 10.000$ & $1 / 500.000$ & $1 / 500.000$ & $1 / 25.000$ & $1 / 25.000$ & $1 / 500.000$ & $1 / 500.000$ \\
\hline & 2 & $1 / 5.000$ & $1 / 2.000$ & $1 / 50.000$ & $1 / 50.000$ & $1 / 2.000$ & $1 / 2.000$ & $1 / 25.000$ & $1 / 50.000$ \\
\hline & 4 & $1 / 1.000$ & $1 / 1.000$ & $1 / 1.000$ & $1 / 5.000$ & $1 / 2.000$ & $1 / 1.000$ & $1 / 5.000$ & $1 / 5.000$ \\
\hline & 6 & $1 / 1.000$ & $1 / 1.000$ & $1 / 1.000$ & $1 / 1.000$ & $1 / 2.000$ & $1 / 1.000$ & $1 / 5.000$ & $1 / 1.000$ \\
\hline & 8 & $1 / 1.000$ & $1 / 1.000$ & $1 / 1.000$ & $1 / 1.000$ & $1 / 2.000$ & $1 / 1.000$ & $1 / 5.000$ & $1 / 1.000$ \\
\hline
\end{tabular}

Fonte: Os autores (2020).

\section{DISCUSSÃO E ANÁLISE}

Nota-se para ambos os voos uma grande diferença entre as escalas definidas utilizando o critério do PEC e as escalas definidas utilizando o critério do EP para os casos com 0 e 2 GCP. Nota-se também que houve uma variação abrupta de escalas definidas de acordo com os critérios do PEC Altimétrico para os casos de 6 e de 8 GCP, entre o Voo A (Escala 1/1.000) e o Voo B (Escala 1/5.000).

Em casos onde a escala permitida de acordo com os critérios do PEC diverge da escala permitida de acordo com os critérios do EP, deve-se prevalecer o critério menos rigoroso. Além disso, quando analisados produtos cartográficos digitais tridimensionais, em casos onde a escala permitida de acordo com os critérios para altimetria elipsoidal diverge da escala permitida de acordo com os critérios para planimetria, também deve-se prevalecer o critério mais rigoroso (menor escala).

Sendo assim, a classificação final dos produtos cartográficos digitais gerados neste estudo, com os critérios PEC e EP para modelos tridimensionais, são a menor das escalas para cada caso, como na Tabela 8.

Tabela 8 - Classificação da Escala (combinando os critérios PEC e EP) para produtos cartográficos digitais tridimensionais com base no número de GCP.

\begin{tabular}{|c|c|c|c|}
\hline & \multirow{3}{*}{ Escala } & Voo A & Voo B \\
\hline & & Planialtimétrica & Planialtimétrica \\
\hline & & PEC e EP & PEC e EP \\
\hline \multirow{5}{*}{ 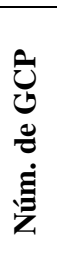 } & 0 & $1 / 500.000$ & $1 / 500.000$ \\
\hline & 2 & $1 / 50.000$ & $1 / 50.000$ \\
\hline & 4 & $1 / 5.000$ & $1 / 5.000$ \\
\hline & 6 & $1 / 1.000$ & $1 / 5.000$ \\
\hline & 8 & $1 / 1.000$ & $1 / 5.000$ \\
\hline
\end{tabular}

Fonte: Os autores (2020).

Como também apontado por trabalhos citados anteriormente (ZANETTI et al., 2017; ALVES JÚNIOR et al., 2018), foi identificado um padrão de aumento na acurácia dos produtos com base no incremento de GCP.

As quantidades de 6 e $8 \mathrm{GCP}$ proporcionaram produtos compatíveis com a escala 1:1.000, classe A para o Voo A, porém esse mesmo número de GCP proporcionou produtos compatíveis com a escala 1:5.000 na classe A quando no Voo B. Atribuímos tal fato ao posicionamento dos dois GCP acrescentados no processamento com 6 GCP (Figuras $5 \mathrm{~d}$ e $6 \mathrm{~d}$ ).

Além disso, destaca-se o fato de que com inserção de apenas 2 GCP, se comparado aos resultados obtidos com $0 \mathrm{GCP}$, a acurácia dos produtos gerados com $0 \mathrm{GCP}$ sofreu grande aumento, passando da Escala 1/500.000 para a Escala 1/50.000 em ambos os Voos.

Observamos uma semelhança para os resultados de classificação dos produtos obtidos pelos Voos A e B no que diz respeito aos casos com 0,2 e 4 GCP, porém quando utilizados 6 e 8 GCP houve grande variação na classificação das escalas.

Tendo em vista que a distribuição dos GCP para os 3 primeiros casos é semelhante e que nos dois últimos casos a maior diferença na distribuição dos GCP se dá ao ponto mais extremo, entendemos que tal disposição contribuiu para o aumento da acurácia do Voo A quando usando 6 e 8 GCP. Diferente do Voo A, onde todos os CP estão "englobados" pelo polígono formado pelos GCP, no Voo B percebemos o 
posicionamento de um CP (2b) localizado no extremo sul da região, sendo este responsável pela maioria das discrepâncias máximas.

\section{CONSIDERAÇÕES FINAIS}

O objetivo principal desse trabalho consistiu em analisar o impacto do número de GCP na acurácia de produtos cartográficos digitais gerados a partir de imagens obtidas por RPA em áreas de terrenos acidentados. Tal objetivo foi alcançado, obtendo como resultado a classificação de dois ortomosaicos e dois DSM, um em escala 1/5.000 e outro como escala 1/1.000 segundo utilizando as diretrizes PEC-PCD/EP.

Apesar de gerados a partir de um mesmo grupo de imagens capturadas em uma área de 0.51 ha com relevo acidentado, a diferença no posicionamento dos GCP resultou em uma variação na escala dos produtos. Além disso notou-se a importância do posicionamento dos CP.

A partir dos resultados obtidos concluímos que além da quantidade de GCP, a disposição dos mesmos é igualmente importante, buscando garantir o posicionamento dos GCP nos bordos da área de estudo, e dos CP na região interna aos GCP.

\section{Agradecimentos}

Esse estudo foi parcialmente financiado pelo CNPq (Conselho Nacional de Desenvolvimento Científico e Tecnológico) por meio de bolsa de mestrado através do processo de número 132614/2018-9 Chamada/Edital GM/GD - Cotas do Programa de Pós-Graduação.

\section{Contribuição dos Autores}

Neste artigo, os autores contribuiram da seguinte maneira: Henrique Lopes Siqueira -: conceptualização, curadoria de dados, investigação, metodologia, visualização, redação - minuta inicial, redação - revisão e edição; José Marcato Junior - conceptualização, análise formal, investigação, administração do projeto, redação - revisão e edição; Edson Takashi Matsubara - supervisão, redação - revisão e edição; Reinaldo Almeida Colares - recursos, redação - revisão e edição; Fabio Martins Santos - recursos.

\section{Conflitos de Interesse}

Os autores declaram que não há conflito de interesse.

\section{Referências}

ALVES JÚNIOR, L. R.; FERREIRA, M. E.; CÔRTES, J. B. R.; CASTRO JORGE, L. A. High accuracy mapping with cartographic assessment for a fixed-wing remotely piloted aircraft system. Journal of Applied Remote Sensing, vol. 12, n.1, 2018.

BRASIL. Ministério da Defesa. Especificação Técnica para a Aquisição de Dados Geoespaciais Vetoriais (ET-ADGV): EB 10-N-72.001, 2a ed. Brasil, 2011.

CAMPOS, M. B., TOMMASELLI, A. M. G., MORAES, M. V. A., JUNIOR, J. M. Análise comparativa dos resultados obtidos pelos métodos de calibração de campo tridimensional e bidimensional. BCG - Boletim de Ciências Geodésicas, v. 21, n. 2, 2015.

COSTA, G. C.; SILVA, D. C. Classificação de mapeamento aerofotogramétrico com imagens de câmeras digitais não-métricas para fins de projetos de estradas. Revista Brasileira de Cartografia, vol. 61, n. 3, 2009.

DEBIASI, P.; MITISHITA, E. A. A influência da calibração aérea de uma câmara digital de baixo custo integrada com um sistema LIDAR no processamento de aerotriangulação. Boletim de Ciências Geodésicas, Curitiba, vol.19, n.4, 687-710, 2013. 
DJI (2017) - Phantom4 Pro/Pro+ User Manual (v1.2). Disponível em: <https://www.dji.com/br/phantom-4pro/info>. Acesso em: 2 abr. 2019.

GINDRAUX, S.; BOESCH, R.; FARINOTTI, D. Accuracy assessment of digital surface models from Unmanned Aerial Vehicles' imagery on glaciers. Remote Sensing, vol.9, n.186, 1-15, 2017

JAMES, M. R.; ROBSON, S.; D’OLEIRE-OLTMANNS, S.; NIETHAMMER, U. Optimising UAV topographic surveys processed with structure-from-motion: Ground control quality, quantity and bundle adjustment. Geomorphology, vol.280, 51-66, 2017.

KRŠÁK, B.; BLIŠŤAN, P.; PAULIKOVÁ, A.; PUŠKÁROVÁ, P.; KOVANIČ, L.; PALKOVÁ, J.; ZELIZŇAKOVÁ, V. Use of low-cost UAV photogrammetry to analyze the accuracy of a digital elevation model in a case study. Measurement, vol.91, 276-287, 2016.

MOLNAR, A.; DOMOZI, Z. Some Practical Problems of Photogrammetry Based Volume Control of Openpit Mines. IEEE 16th World Symposium on Applied Machine Intelligence and Informatics, SAMI 2018. Košice, Herl'any, Slovakia, 2018, 217-224.

NADOLINETS, L.; LEVIN, E.; AKHMEDOV, D. Surveying Instruments and Technology. CRC Press, Inc., United States, 2017.

OLIVEIRA, D. R.; CICERELLI, R. E.; ALMEIDA, T.; MAROTTA, G. S. Geração de modelo digital do terreno a partir de imagens obtidas por veículo aéreo não tripulado. Revista Brasileira de Cartografia, Brasil, vol.69, n.6, 1143-1151, 2017.

PIX4D. Pix4Dmapper. Versão 4.1.23. Pix4D, 2018.

QGIS Development Team. QGIS. Versão 2.18.24 [Las Palmas]. QGIS Development Team, 2019.

RACZYNSKI, R. J. Accuracy analysis of products obtained from UAV-borne photogrammetry influenced by various flight parameters. 2017. 71p. Master Thesis. - Norwegian University of Science and Technology. Department of Civil and Environmental Engineering, Noruega, 2017.

Trimble (2013) - Trimble R8, R6 and R4 User Guide Version 4.80 Revision A. Disponível em: <https://www.trimble.com/support_trl.aspx?Nav=Collection-65944\&pt=Trimble\%20R4>. Acesso em: 2 abr. 2019.

WESTOBY, M. J.; BRASINGTON, J.; GLASSER, N. F.; HAMBREY, M. J.; REYNOLDS, J. M. “Structurefrom-Motion" photogrammetry: A low-cost, effective tool for geoscience applications. Geomorphology, vol.179, 300-314, 2012.

ZANETTI, J.; JUNIOR, J. G.; SANTOS, A. P. Influência do número e distribuição de pontos de controle em ortofotos geradas a partir de um levantamento por VANT. Revista Brasileira de Cartografia, vol.69, n.2, 263-277, 2017.

ZANETTI, J.; PAULA, R. M.; SANTOS, A. P.; MEDEIROS, N. G. Avaliação da acurácia posicional planimétrica de ortoimagens disponibilizadas nos sistemas de informações. Revista Brasileira de Cartografia, vol.68, n.7, 1341-1352, 2016.

\section{Biografia do Autor Principal}

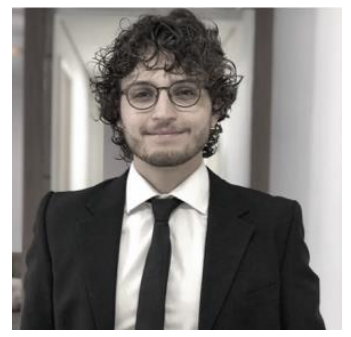

Henrique Lopes Siqueira, natural de Campo Grande/MS (1993). Graduado em Engenharia Civil pela Universidade Federal de Mato Grosso do Sul (UFMS) com período de intercâmbio pelo programa Ciência sem Fronteiras - CAPES na University of Nebraska at Omaha (UNO), realizou seu Mestrado pelo Programa Programa de Pós-Graduação em Tecnologias Ambientais pela UFMS, com enfoque em sensoriamento remoto, desenvolvendo estudos relacionados a aplicação de RPA como ferramenta para cálculo de áreas e volumes. 